Internationale Arbeitsgemeinschaft für Cyanophytenforschung IAC

\title{
4. Symposium über Fragen der Cyanophytensystematik in Kastanienbaum, 1966
}

\section{Verhandlungsbericht}

Von Marianne Pavoni, Zürich

Laut Beschluss am 16. Kongress der Internationalen Vereinigung für Limnologie in Polen (1965) fand vom 2. bis 12 . August 1966 das 4. Symposium der Internationalen Arbeitsgemeinschaft für Cyanophytenforschung (IAC) statt. Unter der Leitung von Herrn Prof. Dr. O. JAAG trafen sich wiederum im Hydrobiologischen Laboratorium der ETH in Kastanienbaum am Vierwaldstättersee (Schweiz) die folgenden Teilnehmer:

Dr. Asta Almestrand, Lund

Dr. K. Anagnostidis, Thessaloniki

Dr. D. Backhaus, Falkau (Schwarzwald)

Dr. E. FJerdingstad, Kopenhagen

Prof. Dr. B. FotT, Prag

Dr. S. Golubić, New Haven

Dr. EDith KanN, Wien

Pıof. Dr. J. Komárek, Třeboñ

Dr. D. Mollenhauer, Frankfurt

Dr. Marianne Pavoni, Zürich

Dr. R. RATHSACK-KÜNZENBACH, Berlin

Prof. Dr. K. Starmach, Krakau

Dr. A. ZEHNDER, Neuenhof AG

Für die Organisation waren Mitarbeiter von Herrn Prof. Dr. O. Jaag, nämlich Dr. H. Ambühl, Dr. Marianne Pavoni, Dr. A. Zehnder, Frl. Beatrix Egri verantwortlich.

12 Kurzreferate bildeten die Grundlage für angeregte Diskussionen, Arbeit am Mikroskop und 2 Exkursionen $\left.{ }^{1}\right) .6$ Referate liegen am Schluss des Verhandlungsberichtes als Veröffentlichungen vor. Da die Diskussionen ganz frei, gleichsam als Gespräch am runden Tisch erfolgten, werden sie hier nicht als Voten, sondern in Form von Zusammenfassungen wiedergegeben.

Eine Gruppe von Beiträgen umfasste grundsätzliche und spezielle Fragen der Blaualgensystematik, eine andere behandelte mehr die Resultate taxonomisch-ökologischer Untersuchungen. Nach diesen Gesichtspunkten sind die folgenden Ausführungen zusammengestellt worden.

1) Es wurden 2 Exkursionen durchgeführt, und zwar, den wichtigsten diskutierten Formenkreisen entsprechend, an Fliessgewässer: eine eintägige an Bäche im Kalkgesteingebiet der Zentralschweiz (Engelberg) und eine zweitägige an Gewässer mit saurer Gesteinsunterlage im Kanton Tessin. 


\section{Grundsätzliche und spezielle Fragen zur Blaualgensystematik}

\section{STARMACH, K.: Zur Taxonomie der Chroococcales (Seite 172)}

In den vergangenen Jahren bemühte man sich wieder in vermehrtem Masse, Unklarheiten der verschiedenen taxonomischen Einheiten zu beseitigen. Dies kommt in der Revision von Arten, Gattungen und sogar Reihen zum Ausdruck. Besonders unbefriedigend ist die Systematik der Chroococcales, die von verschiedenen Seiten neu aufgestellt wurde. Das System der Russen (ELENKIN) kann der Referent wegen seiner zu starken Aufgliederung nicht vollständig anerkennen, obgleich er sich in einigen Punkten daran anlehnt. Noch weniger spricht ihn die starke Reduktion der Gattungen im System von Drouet und DaIry an. Demgegenüber erläutert er das System, wie er es im neu erschienenen polnischen Blaualgen-Bestimmungswerk zur Darstellung gebracht hat.

Da viele Gattungen der Chroococcales sich durch ihre unterschiedlichen Teilungsrichtungen voneinander unterscheiden, kann dieses charakteristische Merkmal taxonomisch ausgewertet werden. Die Chroococcales können daher in die 3 Unterreihen mit eindimensionaler (Coccobactrinales), mit zweidimensionaler (Planimetrales) und mit dreidimensionaler (Stereometrinales) Teilungsrichtung gegliedert werden.

Diskussion. Die Einteilung der Chroococcales auf Grund ihrer verschiedenen Teilungsrichtungen wäre, konsequent durchgeführt, sehr zu begrüssen.

Weniger befriedigend wurde die Wahl der Reihen- und z. T. Familienbenennungen betrachtet, da die Nomenklaturregeln nicht gestatten, Reihen, Familien usw. umzubenennen. Im allgemeinen werden die Familien nach Gattungen benannt. Es besteht aber keine Gattung Coccobactrum, so dass es besser wäre, die Bezeichnung Coccobactraceae durch andere, z. B. Synechocystaceae, zu ersetzen.

Im weiteren versuchte man, die noch immer bestehenden Lücken in der Taxonomie der Chroococcales herauszuarbeiten und am Beispiel einiger Gattungen zu beleuchten. Die Gattungsmerkmale sind oft ungenügend beschrieben, so dass ein und dieselbe Gattung Arten enthalten kann, die eigentlich verschiedenen Gattungen zuzuschreiben sind. Bei anderen Gattungen wie Marssoniella und Tetrapedia stellte es sich heraus, dass es sich gar nicht um Gattungen von Blaualgen handelt.

Eine Gattungs- und Artenrevision ist notwendig. Dazu aber fehlt primär noch immer ein Ausgangswerk der Chroococcales, worauf die neuen Untersuchungen aufgebaut werden könnten. Wohl denkt man verschiedentlich an das Algenwerk von Lemmermann. Doch werden darüber Bedenken geäussert, da die Beschreibung der Arten oft ungenügend ist.

Aber auch ohne Ausgangswerk kann das System kritisch überarbeitet werden, allerdings nur in Form von Untersuchungen an zahlreichen Materialien, was das nächste Referat anhand von 2 Gattungsmerkmalen zeigte.

Golubić, S. : Zwei wichtige Merkmale zur Abgrenzung der Blaualgengattungen (S. 176)

Auf Grund statistischer Methoden wurde die Art und Weise der Zellteilung und Gallertausscheidung bei mehreren z. T. umstrittenen Blaualgengattungen verfolgt. Die graphischen Darstellungen der Messresultate ergaben charakteristische Kurvenbilder, in denen je nach Gattung ein anderer Rhythmus zwischen Wachstum, Teilung und oft auch der Gallertausscheidung zum Ausdruck kommt. Die Beispiele zeigen 
eindrücklich, wie grundlegend wichtig Zellteilung und Wachstumsweise zur Abgrenzung von taxonomischen Einheiten, speziell der Gattungen, sind.

Diskussion. Welches sind gute Kriterien zur Abgrenzung der Gattungen? Diese Diskussionsfrage konnte nicht nur durch gegenseitigen Gedankenaustausch beantwortet werden, sondern verlangt nach weiteren eingehenden Untersuchungen, wie im Beispiel der Gloeocapsa-Chroococcus-Abgrenzung und anderen, wo der Referent die Vermehrung als grundlegendes Gattungsmerkmal dargestellt hatte. Solche Untersuchungen können klare Beweise erbringen, ob taxonomische Revisionen, z. B. im Sinne von Gattungsvereinigungen, allzu grosszïgig durchgeführt worden sind, wie in der Revision von ELENkIN, der Gloeocapsa und Chroococcus zusammengefasst hat, oder noch extremer in der radikalen Revision von DRovET und DAILY. Sicher ist es oft notwendig, mehrere Gattungen zusammenzuziehen, besonders wenn auf Grund ökologischer Untersuchungen die Plastizität der Gattungen festgestellt werden kann.

Wenn man sich einig war, dass die Vermehrung ein gutes Gattungsmerkmal darstellt, so traf dies nicht für die Scheidenausbildung zu, die teilweise als solches abgelehnt wurde. Doch beruht die Abgrenzung der Gattungen Oscillatoria, Phormidium und Lyngbya darauf. Der Hinweis auf dieses Merkmal, das offensichtlich gründlicherer Untersuchungen an zahlreichen Materialien in situ und in vitro bedarf, zeigt, wie wenig sicher die Gattungsmerkmale z. T. noch sind, auch wenn sie sich zur Hauptsache als praktisch brauchbar erwiesen haben. Auch das Herausarbeiten guter Artmerkmale bildet eine wichtige Voraussetzung für eine Systemrevision. Dies wurde aus den nachstehenden Ausführungen ersichtlich.

\section{RathSaCK-Künzenbach, R.: Ist die Drehrichtung ein Artmerkmal? (Seite 185)}

Die Familie der Oscillatoriaceen zeigt neben einer grossen Formenfülle einen einfachen Thallusaufbau, wodurch die Systematik ausserordentlich erschwert wird. Man sieht sich immer wieder genötigt, nach weiteren Bestimmungsmerkmalen zu suchen. Ein solches ist zum Beispiel die Drehrichtung der Fäden, insbesondere bei der Gattung Oscillatoria. Sie wird in der Beschreibung der Arten oft angegeben.

Mit dem allgemein bekannten Färbungsmittel Akridinorange für Kernsubstanz konnte bei den geprüften Oscillatorien eine Übereinstimmung im Verlauf der angefärbten Längsstruktur (DNS des Zentroplasmas) mit der Drehrichtung festgestellt werden.

Diese Beobachtungen lassen vermuten, dass eine engere Beziehung zwischen Längsstruktur und Drehrichtung besteht. Somit wäre die Drehrichtung als genetisch fixiert und als ein Artmerkmal zu betrachten. Sollte daher die Drehrichtung bei Oscillatoria nicht als solches angegeben werden?

Diskussion. Der Beitrag fand die allgemeine Zustimmung. Es wurde von seiten der Teilnehmer bestätigt, dass sich die Drehrichtung als nützliches Bestimmungsmerkmal erweist. So können die beiden Arten Oscillatoria animalis und O. acuminata auf Grund der Drehrichtung auseinandergehalten werden: Oscillatoria animalis dreht nach links, $O$. acuminata nach rechts.

Die relativ kleine Zahl morphologischer Merkmale bei Blaualgen erschwert eine klare Taxonomie. Dies bestätigen die nächsten 5 Referate anhand spezieller Beispiele von Reihen, Gattungen und Arten. 
AnAGnostidis, K.: Zur Kenntnis der apochlorotischen Cyanophyten

Eine wenig bekannte und wenig beachtete Blaualgenreihe, die Pelonematales, umfasst die farblosen Blaualgen, die früher unter den Bakterien eingereiht waren. Die Organismen findet man meist im Hypolimnion von Seen in bodennahen Schichten, vergesellschaftet mit anderen Mikroorganismen, teils thiophilen, teils ferrophilen und anaeroben Charakters. Angereichert und gefärbt auf Membranfiltern, können diese Algen ausgezeichnet beobachtet und zugleich als «Herbarmaterial» aufbewahrt werden.

Diskussion. Diese Reihe, erst heute vor allem durch die Neubearbeitung von SKUJA wieder aktuell geworden, enthält einige Formen, die schon von LAUTERBorN (Die sapropelische Lebewelt) im Jahre 1915 festgehalten und beschrieben worden sind. Vermutlich blieben diese farblosen Blaualgen lange Zeit unentdeckt, da ihre Einordnung infolge ihrer meist äusserst feinen Fäden Mühe bereitet und ein sicherer Entscheid, ob Blaualgen oder Bakterien vorliegen, auch heute in vielen Fällen nicht vollständig abgeklärt ist.

\section{KANN, E.: Bemerkungen zur Gattung Homoeothrix ${ }^{2}$ )}

Der Vergleich von Homoeothrix varians aus dem Seebach bei Lunz (Niederösterreich) mit Homoeothrix janthina aus der Tschechoslowakei hat gezeigt, dass es sich höchstwahrscheinlich um die gleiche Art handelt, der dann aus Prioritätsgründen die Bezeichnung Homoeothrix janthina zu geben wäre.

Ferner sind mehrere Arten von Homoeothrix, die im Lunzer Seebach und anderen Bächen vorkommen, nicht zu bestimmen. In Übereinstimmung mit KOMÁREK könnte es sich um neue Arten handeln. Eine Revision der Gattung Homoeothrix ist dringend notwendig und soll von der Referentin in Zusammenarbeit mit KoMÁREK in den nächsten Jahren erfolgen. Die endgültige Benennung der erwähnten Arten soll später festgelegt werden.

Diskussion. Die kurze Mitteilung über die Gattung Homoeothrix ergab eine lebhafte Diskussion über den Formenkreis der Rivulariaceen ohne Heterozysten. Leptochaete, Amphithrix und Tapinothrix sind nachgewiesenermassen schlecht beschriebene Gattungen. Einzig Homoeothrix, deren Namen als "nomen conservandum» vorgeschlagen worden ist (KOMÁREK), kann als gute Gattung betrachtet werden.

ANAGNostidis, K., und RATHSACK-KüNZENBACH, R. : Ist Isocystis pallida eine Blaualge oder ein hefeartiger Pilz? (Seite 191)

Die Gattung Isocystis ist hinsichtlich ihrer systematischen Stellung sehr umstritten. Sie wurde schon unter den Nostocaceen, dann wieder unter den Oscillatoriaceen und zuletzt unter den Pseudonostocaceen eingeordnet. Besondere Schwierigkeiten bereitet aber die Art Isocystis pallida, eine euryvalente Thermalalge, die weiter verbreitet ist als man bis anhin angenommen hatte. Sie weist auf den ersten Blick neben reinen Blaualgenmerkmalen solche, die an einen hefeartigen Pilz erinnern, auf. Die einen Autoren betrachten sie deshalb als zu den Blaualgen, die anderen als zu den Pilzen (Gattung Torulopsidosira) gehörig.

Auf Grund von Materialien aus verschiedenen Thermalquellen und bestimmten Untersuchungsmethoden versuchte man die Taxonomie dieser Art näher abzuklären.

2) Vorläufiger Beitrag; wird später veröffentlicht. 
Die Resultate dieser Untersuchungen und Material von Isocystis pallida wurden den Symposiumsteilnehmern zur kritischen Beurteilung vorgelegt.

Diskussion. Bei der mikroskopischen Betrachtung erhielten die Teilnehmer den Eindruck, dass es sich um eine Blaualge handelt. Zur Klärung der allerletzten Unsicherheiten wären zusätzliche Untersuchungsmethoden notwendig. Mit Hilfe des Elektronenmikroskops müssten Chlorophyllamellen nachgewiesen werden können, die bei Torulopsidosira fehlen müssten. In den Isocystiszellen hingegen müsste ein Kern fehlen, während in den Torulopsidosirazellen ein Kern nachzuweisen wäre. Zudem wären Klonkulturen sehr erwünscht, um die physiologischen Eigenschaften dieser Art bestimmter zu erfassen.

Es wurde vorgeschlagen, die umstrittene Isocystis pallida bis zur sicheren Abklärung der diskutierten Merkmale bei ihrem Namen zu belassen. Dadurch können weitere Verwirrungen vermieden werden.

Besondere Schwierigkeiten beim Bestimmen von Blaualgen treten nicht nur auf, weil oft wenig Merkmale zur Verfügung stehen, sondern weil diese zudem noch stark variieren können. Allzu leicht werden dann neue Varietäten beschrieben. Dass in solchen Fällen die statistische Auswertung der Messresultate ganz besonders angebracht ist und wertvolle Resultate erbringt, zeigte das nächste Referat.

\section{Almestrand, A.: Taxonomische Studien in Oxydationsteichen (Seite 199)}

Oxydationsteiche in Südschweden wurden speziell auf Blaualgen untersucht. Eine der häufigsten Arten, die oft massenhaft auftrat, war Oscillatoria tenuis. Sie zeichnet sich aus durch die grosse Variabilität der Trichombreite $(3,5-10 \mu)$.

Gestiitzt auf statistische Messungen zahlreicher Populationen kam die Referentin zum Ergebnis, dass zwar durch die unterschiedlichen Trichombreiten Mikrotaxa oder Kleinformen entstehen, der Unterschied ihrer Trichombreiten jedoch nicht signifikant ist, um neue taxonomische Einheiten aufzustellen. Auch die Varietäten $O$. tenuis var. natans und var. tergestina sind dem Resultat der statistischen Auswertungen entsprechend nur als solche Kleinformen zu betrachten und in die Hauptform einzuziehen. Oscillatoria tenuis stellt demnach nicht, wie zu vermuten wäre, eine Sammelart, die bei genauerer Untersuchung in viele niedere taxonomische Einheiten aufgegliedert werden könnte, dar. Sie umfasst nach ANAGNostidis' Bezeichnung einen Formenschwarm von systematisch nicht trennbaren Mikrotaxa.

Diskussion. Das Schwergewicht der Diskussion lag auf der Frage, wie die Entstehung solcher Formenschwärme zu erklären ist. Eine auffallend starke Aufsplitterung in Kleinarten findet man im ganzen Organismenreich dort, wo ungeschlechtliche Fortpflanzung existiert. Zudem gibt es Organismen, die leicht mutieren, andere, die in dieser Beziehung stabil sind. Liegt eine leicht mutierende Form von ungeschlechtlicher Fortpflanzung vor, wie es z. B. bei Blaualgen der Fall sein kann, so steigt die Zahl der Kleintaxa schnell an und es entsteht ein Formenschwarm, bei dem dann unter anderen das Merkmal der Trichombreite - ein im allgemeinen konstantes Bestimmungsmerkmal der Blaualgen - stark variiert.

$\mathrm{Ob}$ in einer Population die Unterschiede zwischen den einzelnen Individuen genetisch bedingt sind oder ganz einfach durch eine grosse Variationsbreite der entsprechenden Merkmale verursacht werden, ist bis heute bei Blaualgen nur in wenigen 
Fällen abgeklärt worden. Mittels Klonkulturen kann diese Frage beantwortet werden.

Die Diskussion ergab, dass speziell bei der Bearbeitung von Merkmalen, die eine grosse Variationsbreite aufweisen, sowohl die statistische Auswertung von Messresultaten als ganz besonders die Herstellung von Klonkulturen zu empfehlen ist.

KANN, E.: Beobachtungen und Bemerkungen zu den Arten Chamaesiphon incrustans und Chamaesiphon curvatus $^{3}$ )

Die eindeutige Bestimmung von Chamaesiphon incrustans und Chamaesiphon curvatus ist oft schwierig, da beide Arten ähnliche Entwicklungsstadien aufweisen. Als wesentliche Unterscheidungsmerkmale der genannten Arten gelten die Grösse der Sporangien sowie die Zahl der abgeschnürten Exosporen.

Langjährige Untersuchungen dieser Arten aus verschiedenen Biotopen führten zu folgenden Beobachtungen und Resultaten:

Die als Ch. incrustans benannte Chamaesiphonart kommt in reichlicher Menge als Epiphyt auf Fontinalisblättern im kalkreichen, sommerkalten Mittersee bei Lunz (Niederösterreich) vor, der sogenannte Ch. curvatus in ebenso reichlicher Menge auf Fontinalisblättern des Seebaches, der den Abfluss des Mittersees bildet und ebenfalls sommerkalt ist.

Während Ch. incrustans im Mittersee in 1-11/2 $\mathrm{m}$ Tiefe im ruhigen Wasser auftritt, ist Ch. curvatus in stark strömendem Wasser zu finden.

Im Seebach sind meistens Exemplare mit den für Ch. curvatus charakteristischen zahlreichen Exosporen entwickelt, daneben aber auch solche, die in der Grösse der Sporangien sowie in der Zahl der Exosporen (1-3) mit Ch. incrustans übereinstimmen. Beim sogenannten $C h$. incrustans aus dem Mittersee konnten nie mehr als 1-3 Exosporen beobachtet werden.

Die Referentin hegt die Vermutung, dass der sogenannte Ch. incrustans, der im ruhigen Wasser wenige Exosporen bildet, durch die Bewegung des Wassers und damit im physiologischen Sinne durch erhöhte Nahrungszufuhr zu intensiverem Wachstum und erhöhter Exosporenabschnürung angeregt wird, so dass Stadien entstehen, die mit dem sogenannten $C h$. curvatus übereinstimmen. Demnach könnte $C h$. curvatus als ein Ökotypus von Ch. incrustans aufgefasst werden.

Im Eulitoral des Traunsees in der Wellenschlagzone wurde der sogenannte $C h$. curvatus in typischer Ausbildung als Epiphyt auf Cladophora gefunden. Zugleich waren diese Fundstellen durch häusliche Abwasser stärker verunreinigt. Wäre es demnach nicht denkbar, dass eine gewisse Eutrophierung des Wassers auf das Wachstum und die Exosporenabschnürung anregend wirkt? Dieselbe Frage taucht auf, wenn in der Literatur Ch.curvatus mehrfach aus stehenden Gewässern, vor allem auch aus Teichen erwähnt ist.

In weiteren vergleichenden Untersuchungen in situ und in vitro wird man die Frage, ob mit $C h$. curvatus und $C h$. incrustans nur 2 Erscheinungsformen des gleichen Taxons vorliegen, abzuklären versuchen.

Diskussion. Die Teilnehmer konnten die Feststellungen z. T. bestätigen. In Schwarzwaldbächen trat der typische Ch. incrustans mit wenigen Sporen auf, in

3) Vorläufiger Beitrag; wird später veröffentlicht. 
Bächen des Saargebietes jedoch wurden Exemplare mit bis zu 10 Sporen beobachtet. Ferner wurde erwähnt, dass auf Fontinalis immer nur Ch. incrustans, auf Cladophora Ch. curvatus gefunden worden sei.

Man war sich einig, dass die beiden Arten in typischer Ausbildung klar zu unterscheiden sind, sich in den Jugendstadien aber ähnlich sein können. Ganz allgemein gelangte man zur Überzeugung, dass für die Beurteilung schwieriger Formenkreise die Kenntnis ihrer Merkmale ganz besonders bei optimalen Bedingungen notwendig ist. $Z$ wei unsichere Arten wie $C h$. incrustans und $C h$. curvatus sollten wenn möglich an ein und demselben Standort beobachtet werden können. Parallel zu regional vergleichenden Untersuchungen müsste die Plastizität der fragwürdigen Arten in Kulturen abgeklärt werden.

Dieselbe Gattung kam im nächsten Referat nochmals eingehend zur Sprache. Das Gewicht lag vor allem auf Untersuchungsresultaten, die mit Hilfe der Substratexponierung erzielt worden waren. Der Referent wagte nicht, zum Chamaesiphon-incrustans-Ch.-curvatus-Problem Stellung zu nehmen, da er bei seinen Untersuchungen nur Ch. incrustans und Ch. incrustans var. elongatus gefunden hatte.

\section{Taxonomisch-ökologische Untersuchungen}

Backhaus, D.: Notizen zur Morphologie, Systematik und Ökologie von Chamaesiphonarten in Fliessgewässern (Donauflüsse) (Seite 211)

Auf Ziegelsteinen wurden künstliche Aufwuchsfolien, sogenannte Polyäthylenklarsichtfolien, montiert und maximal 3 Monate in Donauquellflüssen exponiert. Die übliche Expositionszeit der künstlichen Substrate von etwa 30 Tagen war für einige Chamaesiphonarten zu kurz und musste bis auf 3 Monate erweitert werden.

Auf diese Weise beobachtete man die Entwicklung und die morphologisch charakteristischen Merkmale von vier verschiedenen Chamaesiphontaxa: Chamaesiphon fuscus (früher Ch. ferrugineus), Ch. polymorphus, Ch. incrustans und Ch. incrustans var. elongatus.

Die Methode der künstlichen Aufwuchsträger gab einerseits wertvolle Hinweise, um schon in jungen Entwicklungsstadien gewisse Chamaesiphonarten gegeneinander abzugrenzen. Andererseits bestätigte sie von neuem, wie leicht gewisse Arten in jungen Stadien mit anderen zu verwechseln sind, z. B. der junge Ch. fuscus mit Ch. regularis und der junge $C h$. polymorphus mit $C h$. subglobosus.

Die Donauquellbäche bieten in ihrem Verlauf, hervorgerufen durch wiederholten Wechsel des geologischen Untergrundes und durch Abwasserbelastung, unterschiedliche Wasserqualitäten. Innerhalb dieses Milieuspektrums konnten für die genannten Taxa mit Ausnahme von Ch. incrustans Bezirke optimaler Mengenentfaltung abgegrenzt und chemisch näher charakterisiert werden.

Diskussion. Die taxonomischen Beobachtungen der 4 Arten waren so klar und überzeugend dargelegt worden, dass sich die Diskussion mehr auf die Methode der künstlichen Substratexponierung und die Oberflächenbeschaffenheit der Substrate konzentrierte und zu guter Letzt bei ökologischen Fragen erschöpfte. Als günstig erwiesen sich sterile Steine, geritztes Glas; noch besser sind Plastikfolien. Die chemische Beschaffenheit des Substrates hingegen schien bei den Untersuchungen in den 
Donauquellflüssen keinen sichtbaren Einfluss auf die Zusammensetzung und das Wachstum der Algen gehabt zu haben. Es war gleichgültig, ob das Substrat lebend (Pflanzenstengel) oder tot (Holzstücke), ob die Steine aus Kalk oder kalkfrei waren. Solche Feststellungen können natürlich nicht verallgemeinert werden. So konnten im Lago Maggiore im supralitoralen Bereich eindeutig Unterschiede im Bewuchs von Kalk- und Kieselsteinen beobachtet werden, nicht aber in der untergetauchten Zone. Wahrscheinlich spielt hier die Kombination der Umweltfaktoren eine Rolle, das Zusammenwirken von Temperatur, Wasserbewegung, chemischer Zusammensetzung und deren quantitativer Einwirkung.

Die Frage nach dem Einfluss der Umweltfaktoren auf das Auftreten bestimmter Arten ist speziell im Zusammenhang mit der biologischen Beurteilung der Gewässer sehr aktuell. Das anschliessende Referat brachte eine kurze Mitteilung über die Einwirkung verschiedener Konzentrationen von Abwasser auf die Zusammensetzung von Algenbiozönosen.

KanN, E.: Untersuchungen des Aufwuchses auf Steinen in den Fliesswasserrinnen der Versuchsstation Tüffenwies der EAWAG, Zürich ${ }^{4}$ )

Die 4 Fliesswasserrinnen der Versuchsstation Tüffenwies der EAWAG Zürich, von denen 3 Zugaben von $2 \%, 4 \%$ und 10\% mechanisch gereinigtem städtischem Abwasser zu Grundwasser und die vierte reines Grundwasser enthalten, wurden anhand einer einmaligen Probenahme auf ihre Aufwuchsalgen hin untersucht. Spezielle Aufmerksamkeit schenkte man dabei den Cyanophyten. Am Boden der Rinnen liegen Schottersteine, deren Aufwuchs schon makroskopisch feststellbar von Rinne zu Rinne einen anderen Charakter trägt.

Die Steine der Rinne mit 10\% Abwasser zeigten einen dichten grauen Bewuchs von Sphaerotilus natans, ein Abwasserbakterium, das in schwächerem Masse auch in der Rinne mit 4\% Abwasser auftrat. Der Belag der übrigen Rinnen war meist grün bis gelbgrün gefärbt. Was die Cyanophyten anbelangt, war zu beobachten, dass im verunreinigten Wasser die Artenzahl deutlich abnimmt. Im reinen Wasser waren etwa 12 Arten vertreten, im $2 \%$ verunreinigten nur 5 Arten und im 10\%igen nur mehr 3 Arten. Auffallend war jedoch, dass gewisse Arten, und zwar Chamaesiphon polymorphus und erstaunlicherweise auch Scopulonema minor, in allen Rinnen recht üppig vorhanden waren. Man erhielt den Eindruck, dass eine gewisse Erhöhung des Abwassers von Vorteil für die Entwicklung dieser beiden Arten war. Bei einer Konzentration von 10\% Abwasser trat als bekannter Schmutzwasseranzeiger auch Phormidium foveolarum auf.

Im Gegensatz zu den Cyanophyten waren im verunreinigten Wasser die Grünalgen stärker entwickelt. In den Rinnen mit 2\% und 4\% Abwasser waren sie häufiger und artenreicher vertreten als im reinen Wasser. Davon war eine Art, Gongrosira de Baryana, im 10\%ig verunreinigten Wasser noch sehr gut entwickelt. Aus der Gruppe der Rhodophyceen wal es Pseudochantransia pygmea, die bei diesem Belastungsgrad noch gut entwickelt war.

Der Aufwuchs in der Rinne mit reinem, kalkreichem Wasser ist sehr ähnlich demjenigen des reinen, kalkreichen Lunzer Seebaches (Niederösterreich), den die

4) Vorläufiger Beitrag; wird später veröffentlicht. 
Referentin seit einigen Jahren untersucht. Die häufigsten Arten dieser Rinne waren: Chamaesiphon polymorphus, Scopulonema minor (= Pleurocapsa minor), Homoeothrix varians, Rivularia haematites. Seltener waren die Arten Chamaesiphon incrustans, Ch. polonicus, Hydrococcus Cesatii (= Oncobyrsa Cesatiana), Phormidium sp., Leptochaete rivularis (= Homoeothrix sp.).

Diskussion: Die knapp gehaltene Mitteilung streifte wiederum den Formenkreis von Chamaesiphon und zusätzlich den von Scopulonema und Homoeothrix, Gattungen, die im Laufe des Symposiums wiederholt diskutiert worden sind.

Zudem zeigt die Diskussion, wie wertvoll Untersuchungen unter genau definierten Umweltbedingungen sind. Sie sollten in vermehrtem Masse durchgeführt werden, ganz besonders heute, wo sich die Gebiete der Taxonomie, Ökologie und angewandten Limnologie durch ihre Resultate gegenseitig bef uchten und ergänzen. Speziell zu empfehlen wären solche Studien an Blaualgen, die ihre Merkmale je nach Umweltbedingungen stark verändern.

Extreme Standorte eignen sich besonders für ökologische Untersuchungen, da die Zahl der Arten und dadurch ihr gegenseitiger Einfluss stark reduziert ist. Zudem kann die Einwirkung der vorherrschenden Faktoren klar erfasst werden.

Golubić, S.: Algenmatten im Salzwasser - ihre taxonomischen und ökologischen Probleme ${ }^{5}$ )

Die flachen kalkreichen Gewässer der Plattform der Florida-Keys südlich der Floridahalbinsel (USA) bieten zahlreiche Biotope für Blaualgen. Davon sind die Algenüberzüge oder Algenmatten der temporären Tümpel der Mangrovenformation im Supralitoral und in den «Rock-pools» der Gezeitenzone näher bearbeitet worden.

Die temporären Tümpel werden vom salzigen Grundwasser und vom Regenwasser gespiesen und unterliegen einem starken Wechsel des Salzgehaltes und einer häufigen Austrocknung. Unter diesen extremen Bedingungen gedeiht eine artenarme Vegetation, bestehend aus Lyngbya aestuarii, Microcoleus chthonoplastes und einer nicht bestimmbaren Hormogonalen, die vielleicht unter der Gattung Hydrocoleum unterzubringen ist. Microcoleus chthonoplastes weist eine Aufsplitterung in morphometrisch unterscheidbare Mikrotaxa auf, die sich im allgemeinen in eine phykoerythrinarme und eine phykoerythrinreiche Gruppe zusammenschliessen lassen.

Die Algenmatten der flachen Gezeitenzonen sind 5-10 $\mathrm{mm}$ dicke, fleischfarbene Überzüge in den Vertiefungen, die während der Ebbe als «Rock-pools» unter Wasser bleiben. Trotz starker Erwärmung und Verdunstung sind die Lebensbedingungen in diesem Biotop nicht so extrem wie in den temporären Tümpeln. Diese Algenmatten zeigen eine vertikale Differenzierung auf. In der oberen Schicht dominieren $A$ phanocapsa mucicola, Spirulina subtilissima und Schizothrix minuta. Etwas tiefer herrscht Lyngbya semiplena, begleitet von Calothrix scopulorum und einigen lithophilen Formen vor. Im unteren Teil dieser Schicht lebt eine dichte Nematodensiedlung. Die untersten Schichten der Algenmatten werden von Purpurschwefelbakterien beherrscht.

In der beschriebenen Matte sind zwei dichte Schichten von $\mathrm{CaCO}_{3}$-Partikeln beobachtet worden. Dieses Material ist allochthon und wird von Stürmen herangefegt

5) Vorläufiger Beitrag; wird später veröffentlicht. 
und von den Algen eingefangen. Die untere Schicht wird zusammengepresst und zementiert. Dieser Vorgang scheint die Folge rhythmischer Schwankungen des $\mathrm{CO}_{2^{-}}$ Druckes in der Biozönose zu sein, die durch einen wiederholten Wechsel zwischen der Lösung und Fällung des Kalkes eine beschleunigte Verfestigung des losen Karbonatsedimentes verursachen. Auf diese Weise scheint sich die erwähnte Biozönose an einer raschen Ausbildung harter Kalkkrusten in der Gezeitenzone der Florida-Keys zu beteiligen.

Diskussion. Taxonomisch interessant ist Microcoleus chthonoplastes, die durch ihre Aufsplitterung in morphometrisch unterscheidbare Mikrotaxa eigentlich einen Formenschwarm darstellt. Es stellte sich die Frage, ob solche Formenschwärme nicht hauptsächlich in Biotopen mit extremen Umweltfaktoren zu finden sind.

Besonders hervorgehoben wurde die Tatsache, dass die Blaualgen durch ihren schnellen Stoffwechsel im warmen Klima Floridas ihre Umwelt enorm beeinflussen, indem sie wesentlich an der Bildung der Kalkkrusten beteiligt sind. Analoge Beispiele finden wir im Süsswasser in Form der «Schröfenbildung" im Untersee (Bodensee).

Mollenhauer, D.: Beobachtungen an Nostoc, insbesondere an Nostoc pruniforme ${ }^{6}$ )

Als Vorstudie zu einer gründlichen Revision aller Spezies wurde zunächst Material von $N$. pruniforme (L.) Agardh ex Bornet et Flahault im Freiland, in zahlreichen Kulturen, flüssig fixiert und als Exsikkat untersucht und mit anderen Arten der Gattung verglichen. Daneben wurde alle dem Verfasser zugängliche Literatur über die in Rede stehende Art erfasst und durchgesehen. Es ergab sich, dass im Untersuchungsgebiet (Schleswig-Holstein, Deutschland) und wahrscheinlich auch in anderen Regionen (Schweden, Dänemark, Masuren) die Alge einen wohl klimatisch bedingten zweijährigen Entwicklungsgang hat, in dem planktische, benthische und epiphythmenische Stadien aufeinanderfolgen. In zahlreichen, statistisch ausgewerteten Messreihen wurden die spezifischen Abmessungen der verschiedenen Zelltypen unter wechselnden Kulturbedingungen und an lebendem Freilandmaterial unterschiedlicher Herkunft ermittelt. Gegenüber den in der Literatur üblichen, meist auf die Angaben BoRnets und Flahaults zurückgehenden Masszahlen ergaben sich dabei kleine Korrekturen. Bei der sorgfältigen morphologischen und ökologischen Analyse des Materials fielen einige bislang nicht beachtete Merkmale auf, deren Berücksichtigung es erlauben wird, die Artdiagnosen dieser schwierigen Gattung schärfer zu fassen. Aus der Durchsicht einer grösseren Zahl von Exsikkaten und Publikationen ergab sich ein erster Eindruck der Verbreitung von $N$. pruniforme.

Diskussion. Man könnte diese konsequent durchgeführten Untersuchungen ein Schulbeispiel einer taxonomischen Revision eines Formenkreises nennen. Klar ging hervor, dass heute für eine Revision rein morphologisch vergleichende Untersuchungen nicht mehr genügen, dagegen die Berücksichtigung anderer Gebiete wie der Physiologie, Ökologie und Entwicklungsgeschichte die Systematik einen Schritt weiterbringt.

b) Einzelheiten dieser Untersuchungen sind der Originalarbeit zu entnehmen, die nächstens in der Nova Hedwigia erscheinen wird. 


\section{Schlussfolgerungen}

Schon allein das rege Interesse der Teilnehmer am 4. Symposium der «Internationalen Arbeitsgemeinschaft für Cyanophytenkunde» (IAC) über Fragen der Cyanophytensystematik zeigt, dass diese wieder in vermehrtem Masse ein aktuelles Forschungsgebiet darstellt.

Seit den vergangenen 3 Symposien sind Fortschritte $\mathrm{zu}$ verzeichnen. Die meisten Referate handelten in irgendeiner Weise von der Revision höherer oder niederer taxonomischer Einheiten, weniger in Form theoretischer Vorschläge als vielmehr wertvoller Resultate von meist jahrelangen Untersuchungen. Sie betrafen hauptsächlich die Gattungen Gloeocapsa, Chroococcus, Chamaesiphon und Homoeothrix, dann aber auch die Gattungen Nostoc und Oscillatoria und die farblosen Vertreter der Reihe der Pelonematales.

Die statistische Methode wurde bei der Auswertung und zur Beurteilung der Resultate vermehrt angewendet. Damit konnten gerade am 4. Symposium wohlfundierte Untersuchungsergebnisse erläutert werden. Auch das Experiment in Form von Kulturen und künstlichen Expositionssubstraten wurde bei Untersuchungen erfolgreich zu Hilfe gezogen.

Die Diskussionen führten zusammenfassend zu folgenden Feststellungen und Schlüssen: Noch immer sind Revisionen der taxonomischen Einheiten dringend notwendig. Sie verlangen in vermehrtem Masse nach gründlichen, oft langwierigen Untersuchungen der Formenkreise unter Berücksichtigung ihrer Morphologie, Physiologie und Ökologie. Schwierig zu entscheiden ist in vielen Fällen, ob artfixierte oder ökotypische Merkmale vorliegen. Eindeutige Ergebnisse können nur im Experiment, ganz speziell anhand von sorgfältigen Klonkulturen und genau definierten Umweltbedingungen zusammen mit vergleichenden Beobachtungen am natürlichen Standort erzielt werden. Ausserdem ist zur Klärung zytologischer Unterschiede die Methode der Elektronenmikroskopie in der Taxonomie anzuwenden. Alle Resultate sollten wenn notwendig durch statistische Berechnung bewiesen sein.

Nur eine gründliche, intensive Kleinarbeit in diesem Sinne wird dem Forschungsgebiet der Systematik förderlich sein.

Separatdruck aus der Schweiz. Zeitschrift für Hydrologie 29, Fasc. 1 (1967) Herausgegeben mit Unterstützung

der Stiftung der Wirtschaft zur Förderung des Gewässerschutzes in der Schweiz 